\title{
Clinical Evaluation of Targeted Arterial Infusion of Verapamil in the Interventional Chemotherapy of Primary Hepatocellular Carcinoma
}

\author{
Jin Huang • Qiaohong Duan • Pingsheng Fan • \\ Chushu Ji • Yuying Lv $\cdot$ Xinmin Lin · \\ Liting Qian $\cdot$ Xiukun Yu
}

Published online: 21 October 2010

(C) The Author(s) 2010. This article is published with open access at Springerlink.com

\begin{abstract}
This study evaluates the clinical effectiveness of targeted arterial infusion of verapamil in interventional treatment of primary hepatocellular carcinoma. For this purpose, in 273 patients with middle- or late-stage primary hepatocellular carcinoma, verapamil, IL-2, and chemotherapeutic agents were infused into the target tumor vasculature through femoral artery using Seldinger technique. The medications were infused as serial dilutions, and effectiveness was evaluated after two treatment cycles. Among these 273 patients, 76 cases showed clinical cure or significant improvement, 119 cases improved, 64 cases stabilized, while 14 cases progressed or deteriorated. In 238 patients, KPS score and body weights were stabilized. Regarding side effects, 99 patients $(36.3 \%)$ developed leukopenia; 160 patients had gastrointestinal reactions (58.6\%); 80 patients (29.3\%) presented with elevated ALT/ AST profile; and 65 cases (23.8\%) had pyrexia; however, these side effects abated quickly. No elevations in BUN/Cr and/or allergic reactions were observed. Pre- and postintervention cardiac function did not change in all the patients. No significant change was observed in ECG. Liver function was also improved after two cycles of treatment. It was concluded that verapamil management via targeted arterial infusion could effectively reverse the multidrug resistance in cancer cells in primary hepatocellular carcinoma patients and therefore enhanced the efficacy of chemotherapy.
\end{abstract}

J. Huang · Q. Duan · P. Fan $(\bowtie) \cdot$ Y. Lv · X. Yu

Anhui Tumour Hospital, Hefei 230031, China

e-mail: fanpingsheng@csco.org.cn

C. Ji · X. Lin · L. Qian

The Provincial Hospital of Anhui, Hefei 230001, China
Keywords Verapamil - Arterial perfusion - Primary hepatocellular carcinoma - Multiple drug resistance . P-glycoprotein

\section{Introduction}

Primary liver cancer is one of the common malignant tumors, is ranked 6th in cancer incidence rates, and 3rd by death in the world [1]. Presently, surgical excision is the main treatment method with regard to patients with primary liver cancer but about $15 \%$ of patients can be benefited from it in the long run [2]. Given the fact that the onset of liver cancer is more hidden with no obvious symptoms, most patients are diagnosed at the middle or later stages and thus lose the opportunity of a radical surgical treatment. Minimally invasive treatments can have significant therapeutic benefits in prolonging the survival time and improving the quality of life of patients [3]. In this regard, trans-catheter arterial chemoembolization (TACE) is currently the preferred standard approach [4]. Since its first application [5], the technique has been widely used and also further developed. However, the traditional target arterial chemoembolization has limited treatment efficacy as 1-year cumulative survival rate was found to be $58-75 \%$ [6-8] while the long-term survival rate was much lower, perhaps due to development of multidrug resistance (MDR) [9-11]. In vitro studies show that $6-10 \mu \mathrm{moL} / \mathrm{L}$ $(2.94-4.91 \mu \mathrm{g} / \mathrm{mL})$ verapamil completely inhibits MDR of P-gp reversal cancer cells and increases tumor cell sensitivity to chemotherapeutic drugs. However, serious cardiovascular side effects are observed in patients with serum concentrations of $1-2 \mu \mathrm{moL} / \mathrm{L}(0.49-0.98 \mu \mathrm{g} / \mathrm{mL})$. The drug inhibits MDR only as the tissue concentration is $3-10$ 
times the blood concentration $[12,13]$. So far, intravenous verapamil stage-I clinical trials have failed to achieve satisfactory results.

First-pass effect of drugs used in hepatic arterial perfusion (IA) is considered significant in the liver uptake and metabolism. In this concern, first-pass hepatic uptake of fotemustine (FM) at a dose rate of $100 \mathrm{mg} / \mathrm{m}^{2}$ in patients with liver metastases was found to be 0.9 times higher in IA administration as compared with intravenous injection. Thus, the amount of drug received in normal tissue was significantly reduced and, consequently, the side effects were minimized [14]. Following hepatic intra-arterial infusion of fluorouracil (5-FU) at different doses and injection speeds in pigs, the drug could not be measured in either hepatic vein or peripheral blood and was taken up almost entirely by the liver for metabolism [15]. In this study, based on the previous observations in canines [16], the authors sought to determine the chemotherapeutic efficacy and toxicity of verapamil using femoral artery puncture Seldinger technique [17] in patients with primary liver cancer.

\section{Patients and Methods}

\section{Patients}

Two hundred seventy-three hospitalized patients (from October 1999 to May 2009) of primary hepatocellular carcinoma, comprising of 267 males and 6 females, were enrolled in the study. AFP was tested by quantitative chemiluminescence immunoassay (CLIA) before treatment. There were 48 patients with AFP $<20 \mu \mathrm{g} / \mathrm{L}, 45$ patients with $20 \mu \mathrm{g} / \mathrm{L}<\mathrm{AFP} \leq 200 \mu \mathrm{g} / \mathrm{L}, 59$ patients with $200 \mu \mathrm{g} / \mathrm{L}<\mathrm{AFP} \leq 400 \mu \mathrm{g} / \mathrm{L}, \quad$ and 121 patients with AFP $>400 \mu \mathrm{g} / \mathrm{L}$.

\section{Inclusion Criteria}

The diagnosis of primary hepatocellular carcinoma was made according to the clinical diagnostic criteria of National Research Council of China [18]. The following inclusion criteria were used for patient recruitment in the study: Age 18-70 years; KPS score $\geq 70$; Heart rate $>60$ beats per min; Cardiac function 0-1 level; Liver function CHILD classification B or above B; and Life expectancy $>3$ months. The study was approved by the institutional ethics committee of our hospital and informed written consent was obtained from each patient before enrolment in this treatment regimen. Only the patients in whom Seldinger catheter could be passed through the tumor artery were included in this study.

\section{Exclusion Criteria}

The patients were excluded from enrolment in the study under following conditions: a history of surgical intervention of the tumor, history of allergy to iodine medication, pregnancy or breast feeding, mental illness or retardation, acute infections, symptoms of the central nervous system, white blood cell (WBC) count $<4.0 \times 10^{9} / \mathrm{L}$, red blood cell (RBC) count $<10.0 \times 10^{9} / \mathrm{L}$, hemoglobin $(\mathrm{Hb})<60$ $\mathrm{g} / \mathrm{L}$ or any blood coagulation disorders. Also the patients in whom the standard treatment could not be strictly followed for some reasons or those presenting with serious adverse effects or the missing information cases posing difficulty in proper assessment were excluded from the study.

\section{Treatment Regimen and Hepatic Arterial Embolization}

Each patient was managed with intervention therapy for more than two cycles. One cycle was defined as one time intervention treatment per month and at least 2 cycles of intervention treatment were given in each case. The effect of the interventional management was evaluated after 2 months. In all patients, femoral artery was punctured using Seldinger technique. Verapamil, IL-2, Adriamycin (ADM), cisplatin (DDP) and 5-FU were infused into the tumor site via celiac artery. Then the catheter was passed into the right or left hepatic artery and embolization was performed by using Mitomycin C(MMC) plus iodized oil. The perfusion steps used were as follows: an emulsion was made by dissolving verapamil $(15 \mathrm{mg})$, ADM $(40-50 \mathrm{mg} /$ $\mathrm{m}^{2}$ ), DDP $\left(30-50 \mathrm{mg} / \mathrm{m}^{2}\right)$, 5-FU $\left(500-600 \mathrm{mg} / \mathrm{m}^{2}\right)$ in $30 \mathrm{ml}$ of normal saline (NS) and IL-2 (300 IU) and injected into the branches of the hepatic artery distal to the origin of the gastroduodenal artery. After $15 \mathrm{~min}$, verapamil (10 mg) solution in NS was re-infused. In patients with good blood supply, MMC (10-12 mg) emulsion in $1 \mathrm{ml}$ of $40 \%$ iodized oil (Lipiodol) per $\mathrm{cm}$ of the maximum diameter of lesions was injected and the vessels were embolized.

\section{Study Parameters}

The following parameters included the follow up plan: complete blood count (CBC), liver and kidney functions, routine urinalysis and Alpha-fetoprotein (AFP). Electrocardiogram (ECG), ultrasound (US) or computed tomography (CT) was done for the 1 st time before starting the treatment intervention, for the 2 nd time after 30 days, and for the 3rd time after 60 days from the treatment. In the meantime, information regarding medication side effects, changes in signs and symptoms and Karnofsky performance scale (KPS) index were also recorded. After administering 2 cycles of intervention regimens, an assessment of the clinical benefits was performed including 
the generation of new lesions or changes in existing lesions, KPS index, change of body weight and AFP levels. Heart rate and blood pressure were monitored before, during and $5 \mathrm{~min}$ after the infusion of verapamil. In order to observe the effect of verapamil administration on the cardiovascular system during the interventional treatment, not only monitoring of the dynamic ECG during the operation, but also performing and comparing the pre- and post-intervention ECG results were done. In addition, cardiac function was evaluated for any change(s). The patients were followed up for 1-year survival time after the intervention. Toxicities of the anticancer drugs were evaluated according to the National Cancer Institution (NCI) toxicity grade (0-4) criteria [19]. Cardiac function was classified using the New York Heart Association (NYHA) functional classification (I-IV) criteria [20]. Liver functions were evaluated according to Child-Pugh classification scores [21].

\section{Clinical Cure or Significant Improvement}

If, after two cycles of treatment, the tumor either disappeared or was reduced by more than $75 \%$ and iodized, then oil accumulation was uniform with complete occlusion of the tumor vasculature, or residual tumor vessels were found only in the marginal area.

\section{Improvement}

The tumor was reduced by $30-75 \%$ of its initial size with an uneven accumulation of iodized oil such that the iodized oil-filled area accounted for more than half of the whole tumor mass whereas the tumor vasculature was also significantly reduced.

\section{Stability}

The reduction in tumor size was less than $30 \%$ of the initial mass with a patchy accumulation of iodized oil such that the iodized oil-filled area comprised less than half of the whole tumor mass, and there was no significant decrease in tumor blood vessels.

\section{Progression or Deterioration}

The size of tumor mass was increased, and iodized oil was sparsely distributed or displayed poor accumulation such that the oil-filled area accounted for less than one-third of the whole tumor mass. The tumor vasculature was also found to be significantly increased with or without formation of the new arteriovenous (AV) fistulas between the hepatic artery and portal vein [22].

\section{Treatment Efficacy}

The treatment efficacy was evaluated based on CT scan or US results and AFP quantitative measurements 1 month after the second treatment cycle.

\section{Results}

\section{Clinical Outcome}

After two cycles of treatment intervention, the clinical outcome was analyzed. Of 273 patients, 76 cases were clinically cured, 119 cases were significantly improved, 64 cases remained stable, and 14 cases showed disease progression or deterioration (Table 1). In 137 patients, AFP decreased after one course of treatment, and among them, 88 cases showed a more than $50 \%$ reduction in AFP. In 35 patients, AFP dropped after receiving two treatment courses, and 20 of these individuals showed a more than $50 \%$ AFP reduction. Of the 273 patients, 238 cases showed Karnofsky score $>20$ which was maintained over a period of 4 weeks or more, and 166 patients had a more than $7 \%$ weight gain which was also maintained for a period of at least 4 weeks. Two hundred fifty-nine patients were followed up for an observation period of one year, and the 1-year survival rates reached $81.8 \%$.

\section{Side Effects}

The following side effects were recorded in the patients after the treatment intervention. The major side effects (Table 2) observed were as follows: 99 (36.3\%): patients developed leukopenia; $160(58.6 \%)$ patients presented with gastrointestinal reactions; $80(29.3 \%)$ individuals had elevated ALT/AST; and 65 (23.8\%) cases had fever; however, all these side effects quickly subsided. No allergic reactions or elevations in BUN/Cr were observed. In all the cases, no significant change in the cardiac function (Table 3) was observed before and after intervention. Also, no significant

Table 1 Outcome of verapamil arterial infusion in primary hepatocellular carcinoma patients

\begin{tabular}{llllll}
\hline & Clinically cured or significantly improved & Improved & Stable & Progressed or deteriorated & Total \\
\hline No. of cases & 76 & 119 & 64 & 14 & 273 \\
\hline
\end{tabular}


change was found in ECG (Table 4). The liver function (Table 5) improved after two cycles of treatment. After the first and second cycle of treatment, mean AST values were

Table 2 Major side effects in primary hepatocellular carcinoma patients during intervention treatment $(N=273)$

\begin{tabular}{llllll}
\hline & 0 & 1 & 2 & 3 & 4 \\
\hline Leukopenia & 174 & 35 & 28 & 31 & 5 \\
Hb decreased & 233 & 25 & 13 & 2 & 0 \\
Platelet decreased & 212 & 24 & 27 & 9 & 1 \\
GI reactions & 113 & 78 & 55 & 15 & 12 \\
ALT/AST elevation & 193 & 65 & 15 & 0 & 0 \\
BUN/Cr elevation & 273 & 0 & 0 & 0 & 0 \\
Alopecia & 89 & 135 & 49 & 0 & 0 \\
Fever & 208 & 17 & 22 & 26 & 0 \\
Muscle or Joint pain & 240 & 15 & 18 & 0 & 0 \\
Allergic reaction & 273 & 0 & 0 & 0 & 0 \\
\hline
\end{tabular}

The data were analyzed using SPSS13.0 statistical software. The means were compared using two independent samples t-test, proportion and rate differences were determined using chi-square test. $P$-value $<0.05$ was considered as statistically significant

Table 3 Change in cardiac function $(N=273)$

\begin{tabular}{llllll}
\hline Classification & Normal & I & II & III & IV \\
\hline 1 Day before treatment & 260 & 13 & 0 & 0 & 0 \\
30 Days after treatment & 261 & 12 & 0 & 0 & 0 \\
60 Days after treatment & 261 & 12 & 0 & 0 & 0 \\
\hline
\end{tabular}

The cardiac function was classified according to the criteria of New York Heart Association (NYHA). There was no significant change in all 273 cases before and after the treatment intervention $(P>0.05)$

Table 4 ECG results at 4 time points $(N=273)$

\begin{tabular}{lllll}
\hline Indicator & P-R interval & Q-T interval & QRS & Heart rate \\
\hline 0 min & $0.16 \pm 0.03$ & $0.36 \pm 0.03$ & $0.08 \pm 0.01$ & $75 \pm 13$ \\
$5 \mathrm{~min}$ & $0.16 \pm 0.02$ & $0.36 \pm 0.03$ & $0.08 \pm 0.01$ & $75 \pm 12$ \\
$10 \mathrm{~min}$ & $0.16 \pm 0.03$ & $0.36 \pm 0.03$ & $0.08 \pm 0.01$ & $75 \pm 12$ \\
$20 \mathrm{~min}$ & $0.16 \pm 0.03$ & $0.36 \pm 0.02$ & $0.08 \pm 0.01$ & $75 \pm 12$ \\
$50 \mathrm{~min}$ & $0.16 \pm 0.03$ & $0.36 \pm 0.03$ & $0.08 \pm 0.01$ & $75 \pm 12$ \\
\hline
\end{tabular}

Indicated time points represent the duration after verapamil infusion. No significant differences were found as compared with respective means of the 0 min group $(P>0.05)$

Table 5 Number of cases with abnormal liver function results $(N=273)$

\begin{tabular}{lllll}
\hline & AST & ALT & GGT & ALP \\
\hline Pre-intervention & 260 & 266 & 273 & 150 \\
Post-intervention & 30 & 35 & 37 & 21 \\
\hline
\end{tabular}

87.0 and $23.0 \mathrm{U} / \mathrm{L}$, respectively. Similarly, mean ALT values were 98.0 and $19.0 \mathrm{U} / \mathrm{L}$, respectively, and mean ALP values were 289.0 and $47.0 \mathrm{U} / \mathrm{L}$, respectively. Following the first and second treatment cycles, mean GGT values were 122.0 and $37.0 \mathrm{U} / \mathrm{L}$, respectively. All the parameters showed significant differences $(P<0.05)$.

\section{Discussion}

The MDR refers to a drug-induced phenomenon in which targeted cells become simultaneously resistant to multiple medications with different chemical structures and mechanisms of action. MDR is the main culprit for the failure of chemotherapy in the treatment of malignant tumors. Meanwhile, MDR also constitutes one of main reasons as to why the conventional targeted arterial embolization intervention therapy fails in the chemotherapy of primary hepatocellular carcinoma [11, 12].

The P-glycoprotein (P-gp) is a $170-\mathrm{kD}$ glycoprotein which is an ATPase-dependent membrane transport protein, initially found to be present in the membrane of MDR cells, as colchicine was applied to Chinese hamster ovary cells and a decrease in the intracellular drug concentration was detected [23]. P-glycoprotein hydrolyzes ATP to ADP and releases energy, binds to intracellular anti-tumor drug in the presence of magnesium and "pumps out" cytotoxic drug from cells, and hence decreases the intracellular drug concentration, and consequently the cytotoxic effect of anti-tumor drugs; the phenomenon is known as MDR [911]. The membrane transport of $\mathrm{P}$-gp plays a pivotal role in MDR, and hence P-gp-mediated MDR is also known as the typical MDR. The MDR genes were found to be expressed at relatively low levels in both normal and cirrhotic liver tissues. Since MDR gene expression is significantly changed in untreated liver cancer, it indicates the role for an endogenous MDR. However, in post-chemotherapy liver cancer cells, the change in the MDR gene expression is far more significant and thus suggests the role for an acquired MDR [24].

Verapamil is considered as one of the earliest known modulators of the MDR, and it reverses the MDR by inhibiting the P-gp synthesis-hence called as a chemosensitizer. Thus, by suppressing the P-gp gene expression, verapamil inhibits the efflux of anticancer drugs from tumor cells leading to the accumulation of chemotherapeutic agents, and hence an enhanced cytotoxicity and efficacy of chemotherapy [13]. A few in vitro studies report that verapamil suppresses the mRNA levels of MDR genes including transcription levels of $\mathrm{P}$-gp, reverses the MDR phenotype by also inhibiting the activity of PKC which is required for P-gp phosphorylation, and sensitizes tumor cells to chemotherapeutic agents by increasing the affinity 
between the anti-tumor agents and their corresponding intracellular bind sites [11].

In this study, the authors found that verapamil used at a concentration of $6-10 \mu \mathrm{moL} / \mathrm{L}$ completely reversed the MDR in malignant tumor cells by inhibiting P-gp and enhancing sensitivity of tumor cells to chemotherapy. However, when the serum concentration of verapamil reaches 1-2 $\mu \mathrm{moL} / \mathrm{L}$, the cardiovascular system side effects are observed. This is the reason why use of verapamil is limited to clinical applications. The specifically targeted arterial infusion of verapamil increases the tissue drug concentration by 3-10 times the serum drug concentration and hence this approach enables verapamil to reverse MDR while body can well tolerate it [12, 13].

In this study, verapamil, IL-2, ADM, DDP, 5-FU, MMC, and iodized oil were administered in 273 patients through targeted arterial infusion approach. After two cycles of treatment intervention, 76 cases were cured or significantly improved, 119 cases improved, 64 cases stabilized, and 14 cases progressed or deteriorated. In 137 cases, AFP decreased after 1 cycle of treatment while in 35 cases AFP decreased after two treatment cycles. In 238 patients, KPS score and body weight were found to be stabilized, and no significant cardiovascular adverse reactions were observed. We followed 259 patients for an observation period of one year, and the 1-year survival rate was found to be $81.8 \%$ whereas in the previous studies [6-8], the 1-year survival rate after the traditional target arterial chemoembolization was reported as $58-75 \%$. Hence in our study, administration of verapamil by the perfusion approach significantly improved the efficacy of conventional TACE treatment, enhanced the overall survival of patients and did not lead to any significant adverse reactions of the cardiovascular system. The common toxicity complications of grade 3-4 that were observed were almost of the same nature and level as previously reported in the aforementioned studies. However, 14 patients clinically progressed or suffered deterioration. We believe that this failure in improvement in these individuals is attributable, at least in part, to multiple factors such as higher levels of post-therapy leukopenia and severity of gastrointestinal side effects. Therefore, further studies will be required to evaluate in detail the possible influence of these and other similar factors in MDR reversal and overall improvement of hepatocellular carcinoma patients using verapamil as a chemosensitizer. In conclusion, targeted arterial infusion of verapamil via perfusion strategy can reverse the MDR in tumor cells in primary hepatocellular carcinoma patients and increase the effectiveness of chemotherapy.

Acknowledgments We thank Anhui Province Council of Scientific Research for financial support (Grant \# 01023027). We also thank all the participants of the study for their patience and kind cooperation.
Open Access This article is distributed under the terms of the Creative Commons Attribution Noncommercial License which permits any noncommercial use, distribution, and reproduction in any medium, provided the original author(s) and source are credited.

\section{References}

1. Parkin, D. M., Bray, F., Ferlay, J., \& Pisani, P. (2005). Global cancer statistics, 2002. CA: A Cancer Journal for Clinicians, 55, 74-108.

2. Llovet, J. M., Bruix, J., \& Gores, G. J. (2000). Surgical resection versus transplantation for early hepatocellular carcinoma: Clues for the best strategy. Hepatology, 31, 1019-1021.

3. Veltri, A., Moretto, P., Doriguzzi, A., Pagano, E., Carrara, G., \& Gandini, G. (2006). Radiofrequency thermal ablation (RFA) after transarterial chemoembolization (TACE) as a combined therapy for unresectable non-early hepatocellular carcinoma (HCC). European Radiology, 16, 661-669.

4. Vogl, T. J., Naguib, N. N., Nour-Eldin, N. E., Rao, P., Emami, A. H., Zangos, S., et al. (2009). Review on transarterial chemoembolization in hepatocellular carcinoma: Palliative, combined, neoadjuvant, bridging, and symptomatic indications. European Journal of Radiology, 72, 505-516.

5. Goldstein, H. M., Wallace, S., Anderson, J. H., Bree, R. L., \& Gianturco, C. (1976). Transcatheter occlusion of abdominal tumors. Radiology, 120, 539-545.

6. Buijs, M., Vossen, J. A., Frangakis, C., Hong, K., Georgiades, C. S., Chen, Y., et al. (2008). Nonresectable hepatocellular carcinoma: Long-term toxicity in patients treated with transarterial chemoembolization-single-center experience. Radiology, 249, 346-354.

7. Kirchhoff, T. D., Bleck, J. S., Dettmer, A., Chavan, A., Rosenthal, H., Merkesdal, S., et al. (2007). Transarterial chemoembolization using degradable starch microspheres and iodized oil in the treatment of advanced hepatocellular carcinoma: Evaluation of tumor response, toxicity, and survival. Hepatobiliary and Pancreatic Diseases International, 6, 259-266.

8. Liu, Y. M., Qin, H., Wang, C. B., Fang, X. H., \& Ma, Q. Y. (2006). Comparison of therapeutic effectiveness of combined interventional therapy for 1126 cases of primary liver cancer. World Journal of Gastroenterology, 12, 5060-5063.

9. Chen, L., Zuo, G. Q., Wu, J. F., Zeng, G. L., Li, T., Liu, Z. J., et al. (2009). Intercellular transfer of P-glycoprotein in hepatocellular cell line HepG2. Zhonghua Gan Zang Bing Za Zhi [Article in Chinese], 17, 839-842.

10. Yan, F., Wang, X. M., Liu, Z. C., Pan, C., Yuan, S. B., \& Ma, Q. M. (2010). JNK1, JNK2 and JNK3 are involved in P-glycoprotein-mediated multidrug resistance of hepatocellular carcinoma cells. Hepatobiliary and Pancreatic Diseases International, 9, 287-295.

11. Sun, Z., Zhao, Z., Li, G., Dong, S., Huang, Z., Ye, L., et al. (2010). Relevance of two genes in the multidrug resistance of hepatocellular carcinoma: In vivo and clinical studies. Tumori, 96, 90-96.

12. Ford, J. M., \& Hait, W. N. (1990). Pharmacology of drugs that alter multidrug resistance in cancer. Pharmacological Reviews, 42, 155-199.

13. Baumert, C., \& Hilgeroth, A. (2009). Recent advances in the development of P-gp inhibitors. Anti-Cancer Agents in Medicinal Chemistry, 9, 415-436.

14. Fety, R., Lucas, C., Solere, P., Cour, V., \& Vignoud, J. (1992). Hepatic intra-arterial infusion of fotemustine: Pharmacokinetics. Cancer Chemotherapy and Pharmacology, 31, 118-122. 
15. Mori, T., Iwasaki, Y., Takahashi, K., \& Takahashi, T. (1992). Experimental study on adequate 5-FU dose and flow volume for continuous arterial liver infusion. Gan To Kagaku Ryoho [Article in Japanese], 19, 1547-1549.

16. Fan, P., \& Feng, K. (2004). The effect of hepatic artery infusion of verapamil on the canine vital signs and drug blood concentration. J Cancer, 3, 241-244.

17. Miura, T., An, M., Matsumoto, Y., Terakado, H., Murata, M., Takagi, S., et al. (1998). Transcatheter oily chemoembolization and intermittent hepatic artery infusion chemotherapy in the management of advanced hepatocellular carcinoma. Gan To Kagaku Ryoho [Article in Japanese], 25, 1262-1265.

18. Professional Committee of Chinese Anti-Cancer Association of liver cancer. (2001). Clinical diagnostic/staging criteria of primary liver cancer. Zhonghua Gan Zang Bing Za Zhi [In Chinese], 9, 324.

19. Trotti, A., Byhardt, R., Stetz, J., Gwede, C., Corn, B., Fu, K., et al. (2000). Common toxicity criteria: version 2.0, an improved reference for grading the acute effects of cancer treatment: impact on radiotherapy. International Journal of Radiation Oncology, Biology, Physics, 47, 13-47.
20. The Criteria Committee of the New York Heart Association. (1994). Nomenclature and Criteria for Diagnosis of Diseases of the Heart and Great Vessels (9th ed., pp. 253-256). Boston: Mass. Little Brown \& Co.

21. Pugh, R. N. H., Murray-Lyon, I. M., Dawson, J. L., Pietroni, M. C., \& Williams, R. (1973). Transection of the esophagus for bleeding esophageal varises. British Journal of Surgery, 60, $648-652$.

22. Interventional Radiology Group Editorial Board of Chinese Medical Association Radiology. (2001). Interventional therapy for standardization (Draft). The Journal of Radiology, 35, 230-231.

23. Shi, H., Lu, D., Shu, Y., Shi, W., Lu, S., \& Wang, K. (2008). Expression of multidrug resistance-related proteins p-glycoproteinglutathione-s-transferases, topoisomerase-II and lung resistance protein in primary gastric cardiac adenocarcinoma. Cancer Investigation, 26, 344-351.

24. Liu, J., \& Chen, X. (2000). Hepatocellular carcinoma multidrug resistance gene expression and its significance. Journal of Experimental and Clinical Cancer Research, 17, 406-408. 